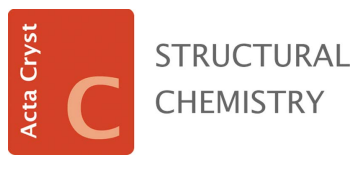

ISSN 2053-2296

istry.
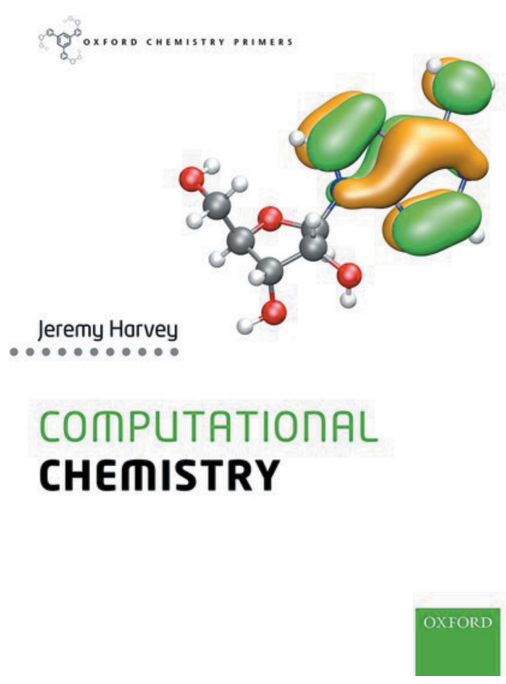

C 2018 International Union of Crystallography

\section{Computational Chemistry. By Jeremy Harvey. Oxford University Press, 2018. Pp. 152. Price GBP 19.99 (paper- back). ISBN 9780198755500}

\author{
Antonio Monari* \\ Université de Lorraine, CNRS, LPCT, F-54000 Nancy, France. *Correspondence e-mail: antonio.monari@univ-lorraine.fr
}

Computational chemistry, as well as the closely related area of molecular simulation techniques, nowadays represents a fundamental tool for the whole of chemical science. Most notably, the field has achieved the capacity to provide answers to complex chemical, or even biological, problems, to be predictive and to complement many experiments. Yet, in many instances, this important role has not fully emerged in undergraduate courses and programmes; as a consequence, textbooks for undergraduate students dealing with molecular modelling and simulations are rare.

It is starting from this consideration that 'Computational Chemistry' by J. Harvey aims at providing a clear and concise, yet as complete as possible, book specifically designed for undergraduate students and covering all the aspects of computational chemistry applications. More specifically, its philosophy is clearly problem-oriented and aims at providing a guide to the practical use of computational chemistry for tackling a given problem, in particular, providing a basic knowledge of the limitations and potentiality of the different methods and the answers they can provide.

More significantly, one of the strengths of the book is the constant use of 'real-life examples' in the form of real calculations performed by the author, which students are encouraged to repeat, to illustrate the different methods and the different problems.

Considering its scope, the main focus of the book is obviously in the application of the different techniques, rather than in an in-depth analysis of the mathematical formalisms. However, this nontrivial task is, in most cases, performed without the loss of rigour and the interested reader will find useful guidance for further development and investigation in the 'Further reading' sections concluding each chapter.

Indeed, the eight chapters of the book cover all aspects of modern molecular modelling and simulation. After a brief introduction (Chapter 1), which is also helpful in making the bridge between computational chemistry and computer science, the author deals with the basics of quantum methods in the determination of the electronic structure of molecular systems, in particular focusing on the Hartree-Fock theory (Chapter 2, Quantum Chemistry). The chapter is remarkable and even some nontrivial concepts, such as the spin contamination for unrestricted methods, are expressed in clear and accessible terms without the loss of rigour, while the crucial role of molecular orbitals in interpreting different chemical properties is underlined. The next chapter (Quantum Chemical Methods) deals with electron correlation in its various forms, from coupled clusters to multi-reference methods, also introducing density functional theory (DFT) calculation. Probably, considering the widespread use of the latter, a more dedicated chapter and a deeper analysis on the performance and limitations of the different classes of functionals would have been welcome.

After having established quantum chemical methods, Chapter 4 deals with the problem of molecular mechanics and, in particular, force field definition. Once again, the chapter provides a very nice formalization of a complex, and in some cases overlooked, problem that is, however, crucial for achieving good accuracy.

Chapters 5 and 6 deal with the definition and exploration of the potential energy surface, either with optimization techniques (Chapter 5) or via molecular dynamics simulation (Chapter 6). The roles of stationary points, minima and transition states are clearly evidenced, together with the proper ways to characterize them.

In a remarkable effort at completeness, Chapter 7 makes the link between computational chemistry and statistical thermodynamics, in particular, providing examples on 
how to retrieve thermodynamic properties, such as free energy, from either the static rigid-rotor approach or via molecular dynamics. This is particularly relevant since free energy is becoming a crucial quantity to be determined whose proper simulation and modelling is in many cases critical and can lead to serious mistakes. However, due to the complexity of the problem, this section is probably less adapted to, or at least will be less immediately understandable by, undergraduate students.

Finally, the last chapter proposes to treat the effects of the molecular environment in multiscale simulation, either via continuum polarizable or hybrid calculations.
'Computational Chemistry' is a very welcome book whose main qualities are the rigour and the completeness achieved without sacrificing the synthesis. This short and easily readable book provides an excellent guide to the world of computational chemistry. It could be successfully used also by graduates, students or researchers from the experimental chemistry fields, who seek a general, yet critical, view of the possibilities offered by modelling and simulation. As such, it is certainly a valuable and productive effort that can strongly benefit the development and the general understanding of computational chemistry and molecular simulation. 